\title{
BILATERAL ATROPHIC LOBAR SGLEROSIS FOLLOWING THROMBOSIS OF THE SUPERIOR LONGITUDINAL SINUS
}

\author{
By \\ R. M. NORMAN, Stoke Park Colony \\ INTRODUGTION
}

Is the category of atrophic lobar sclerosis it is customary to group a number of degenerative affections of the cerebral cortex having a differing and often obscure pathogenesis but a comparatively uniform morphology. Macroscopically, the cortex in these cases shows a shrinkage of the gyri with a corresponding widening of the intervening sulci. Apart from the changes produced by these distorting factors, the general convolutional patterning of the hemispheres is normal, so that usually it is possible to distinguish by naked-eye inspection this type of abnormality from true microgyria of developmental origin. The name 'ulegyria' is often used to describe this condition of atrophy of the convolutions. Microscopically, it is apparent that the neurones of the affected gyri have degenerated to a greater or less degree and that an extravagant proliferation of the fibrous neuroglia is present in those areas where neuronal destruction has been most complete. This gliosis tends to vary according to the time of onset of the primary cortical lesion, being densest and most widespread when the disease has started during fœetal or infantile life and being less pronounced in the more mature brains. ${ }^{15}$ In the latter class of case there is evidence both from the clinical and pathological standpoints that a progressive degeneration of the cortex may occur. In this type of progressive cortical atrophy the lesions are essentially laminar in distribution and affect chiefly the third or pyramidal cell-layer.

As Hallevorden ${ }^{8}$ has pointed out, the similarity of the histopathological findings in cases of lobar sclerosis is no proof of an uniform ætiology. This observation is particularly pertinent when an attempt is being made to evaluate the part played by birth injury in the rtiology of a given case. Although the extensive investigations of Schwartz ${ }^{13}$ have shown that atrophic sclerosis may follow multiple small intracerebral hæmorrhages or softenings, it is clearly unjustifiable to classify all cases of lobar sclerosis with a clinical history of ' difficult labour' as the result of vascular lesions sustained during birth. Moreover, the precise mechanism by which these hæmorrhages are produced is not yet fully understood. According to Schwartz, they 
result from an intense venous engorgement and stasis of blood in the presenting fœtal head, this congestion being brought about by the difference between the atmospheric pressure acting upon the presenting head after rupture of the membranes and the higher intrauterine pressure acting upon the body of the fœetus. This ' suction ' theory has been severely criticized by Rydberg. ${ }^{11}$ In this author's opinion the areas of softening and bleedings commonly found in the brains of stillborn infants are the result of a failure of the arterial blood pressure to compensate for the excessive rise in the general intracranial pressure due to prolonged or sudden compression of the fotal head.

In spite of this conflict of opinion, a valuable outcome of these modern investigations has been to emphasize the importance of an intact vascular supply to the developing cerebrum. This fact is exemplified in the following case of atrophic sclerosis of the cortex associated with obliteration of the superior longitudinal sinus and apparently occurring at the time of birth. Thrombosis of cortical veins, later spreading to the superior longitudinal sinus, was considered by Gowers ${ }^{7}$ to be a common cause of infantile cerebral palsy, but there are few detailed pathological observations to substantiate this view. The case about to be described seems, therefore, to be worthy of record as an example of this type of vascular lesion. Finally, it may be said concerning birth injuries in general and this case in particular that although the clinical history and pathological findings may strongly support the view that the cerebral damage has been produced by the vascular disturbances of parturition, it is an open question whether or not prenatal factors have not been coincidently operative. Individual variation in angioarchitectonics may well play an important but as yet undemonstrable part in the production of these vascular lesions of early life.

\section{GLINIGAL ASPEGT}

Family History. - The patient was the male first born of a family of three, the other two sibs being reported as normal. All three sibs were post-mature. Father and mother were physically and mentally normal and in comfortable financial circumstances. There was no history of nervous disease in the parents or grandparents.

The Pregnancy.-This was of 10 months' duration, and according to the mother labour pains and a partial dilatation of the os uteri occurred exactly four weeks before the child was born. Four months before the birth the mother suffered from an intractable dermatitis, 'like masses of boils,' affecting the thighs and abdomen. A bacteriological examination was made and streptococci only were found. Some months after the birth it was found that the mother was suffering from bad pyorrhoa and an apical abscess of a tooth.

Birth History.-The patient was delivered by head presentation after a dry labour lasting seven hours. Forceps were not used. The child was badly asphyxiated from aspirated meconium and gave hardly any sign of life, but was revived by oxygen and hot and cold baths. No cry was uttered for three hours.

Postnatal IIistory.-There was no rigidity present in the limbs immediately after birth, but no spontaneous movements were made. Twice on the second day the infant went black in the face and breathing was difficult; after this he slept for 15 hours. He was unable to take the breast. By the fourth week signs of stiffness were 
present in the hands ; later this was observed in the arms and then in the feet. Subsequently, in spite of massage and training, no appreciable improvement in the spasticity occurred. Increasing spasticity, especially in the lower limbs, was observed up to about the age of three. No remission or sudden exacerbation of the general condition occurred, and he suffered from no febrile illness during infancy.

Convulsions started when he was about six weeks old, slight limb contractions being associated with a convergent squint and a jerking forward of the head. In the early days these fits were frequent-every two or three hours-but seldom at night. They cccurred on waking after a sleep during the day time. The last fit occurred at the age of nine months.

Dr. H. Cohn of Edinburgh saw the child when he was four years old, and has kindly supplied the following information :

He was poorly nourished, length and weight corresponding to a boy of three years of age. Unable to stand or to speak. The head sank forwards when not supported and when supported there was a marked tendency to turn the head to the side, more often to the right. There was marked salivation and dribbling.

Cranial Nerves.-Except for a slight paresis of the right abducent nerve, eye movements were normal. He could follow a moving finger. The corneal reflexes were present. There was slight paresis of the right side of the face and sometimes involuntary movements of the face produced an expression similar to risus sardonicus. The glossopharyngeal-vagal reflex was diminished and imperfect swallowing sometimes occurred. The tongue showed no paresis, but occasional involuntary movements were observed.

Motor System.-There was marked but variable spasticity of both upper limbs, the tendon-jerks being much increased. Erb's and Hoffman's reflexes of the fingers were present, as was also Tromner's phenomenon. The abdominal and cremasteric reflexes were present on both sides. There was a severe degree of spasticity in the lower limbs, especially in the adductor muscles, so that the right foot usually crossed the left. Knee- and Achilles-jerks were very much increased. No true clonus was obtained. Phenomena of Babinski, Rossolimo, Bechterev and Oppenheim were present on both sides.

Sensation.-Response to pinpricks over the body surface was obtained.

On admission to Stoke Park Colony on December 21, 1934, the patient was aged five years and seven months. His height was 3 feet and he weighed two stone. There was a general brownish pigmentation of the skin and pallor of the mucous membranes. He was unable to stand or sit unaided. He lay in an attitude of general flexion with the legs crossed above the knees. There was a marked rigidity of the upper limbs which to some extent masked the tendon reflexes. The lower limbs also were very rigid and the kneejerks and anklejerks increased, the former being obtained by striking the subcutaneous surfaces of the tibix all the way down the bone. The plantar reflexes were flexor (Babinski's sign negative). The abdominal reflexes were not obtained. There was incontinence of fæces and urine. Since he was speechless, helpless, and unable to make any attempt at simple performance tests he was classified as an idiot.

Terminal Illness.-Five days after admission the temperature rose to $100 \cdot 2^{\circ}$. There was a swelling of the lower lip with an abrasion of the mucous membrane as though the patient had bitten himself. On the evening of the next day the patient suddenly collapsed, the colour being very poor and the breathing distressed. He vomited a little dark brown fluid. On examination he was found to be in a comatose condition. There was bilateral divergent strabismus, pinpoint pupils, and absent terdon-reflexes. Corneal reflexes were present. There was no rigidity of neck muscles. The heart rate was rapid (120) and the sounds pcor. The urine contained a trace of albumen. Later the temperature rose to $103 \cdot 4^{\circ}$ and he died the same evening. 


\section{COMMENT}

So far as the history of this case throws light upon probable causative factors, it would seem that the most significant fact is the attack of respiratory distress and subsequent somnolence that occurred on the sccond day after birth. It is this piece of information, rather than the history cf dry labour and asphyxiation, that is suggestive of some cerebral vascular lesion resulting in bleeding or softening. Rydberg has emphasized the importance of this type of history in cases of fatal birth injury. He made a careful examination of the brains of 75 infants who died within 10 days of birth. He found 34 in which considerable intracranial bleedings or softenings had occurred. All these fatal cases showed a clinical picture which is "perhaps not pathognomonic of intracranial bleedings and primary injuries to the brain substance but is very nearly so. It is characteristic of such cases that there is a symptomfree interval in the first hours or days after birth, that the children fall ill rather suddenly and that then the symptoms of cerebral irritation play an important part during the acute stage of illness. In this group are placed (1) cases which show cerebral motor symptoms such as twitchings, spasms and pronounced general rigidity, and (2) cases which do not present any motor symptoms if the child exhibits a disturbance in the breathing, cyanosis. or a pronounced drowsiness, and a clearly increased tension of the large fontanelle is observed at the same time.'

While such intracerebral bleedings and softenings have evidently a highly lethal effect, some of the individuals in whom they occur must survive. and it is a matter of importance to distinguish this class of case from patients whose spastic paralysis is the outcome of cerebral agenesia. A mere history of difficult labour is not, of course, a sufficient reason for attributing the cause of the diplegia or mental defect to birth injury. Several investigations. notably that of Dayton, ${ }^{5}$ have shown that the prognosis for children born after abnormal labour differs little from that for normal birth. A history of difficult labour is, in fact, common; on the other hand, infantile cerebral palsies are rare. The important point in evaluating a history of birth injury is not the mere fact of abnormal parturition but the history of the characteristic signs of intracranial injury.

There is, therefore, in the present case clinical evidence which suggests that some vascular lesion occurred at or shortly after birth.

\section{MORBID ANATOMY}

Post-mortem Examination.--Immediately after death 10 per cent. formolsaline solution was injected into the skull through the orbits and the cisterna magna, as much cerebrospinal fluid as possible having been previously withdrawn. An autopsy was made about 12 hours after death. The only abnormalities found were the following: The lungs showed congestion of the posterior part of the lower lobes. The skullcap was thin and poorly ossified. 
The dura mater was immovably adherent to the parietal bones and in this situation the lumen of the superior longitudinal sinus was obliterated by dense adhesions. No signs of old hæmorrhages were present. The pia arachnoid membranes over the vertex of the brain were thickened and pinkish in appearance, but not adherent to the cortex. The vessels were very engorged.

The brain and spinal cord were removed and suspended in 10 per cent. formol-saline, representative blocks having been taken for fixation in formolammonium bromide solution.

Macroscopic examination of the brain after stripping the meninges showed that a condition of atrophic sclerosis was present in the gyri of the convexity adjoining the left paracentral lobule. The upper half of the left precentral, the upper third of the postcentral gyrus, and the part of the superior parietal lobule adjacent to the postcentral sulcus were markedly shrunken. The superficial puckering of these gyri gave the appearance of verrucous atrophy. This sclerosed region was not uniformly hard to the touch, for the superficial tissue was soft and friable compared with the increased density of the subjacent parts. This peculiarity was especially noticeable when fresh blocks were being cut out for formol-bromide fixation, and it was found that this deepseated toughness extended in the white matter over a much larger area than might have been supposed from the surface extent of the shrunken gyri. The right hemisphere was macroscopically less affected. Here the area most involved was the postcentral gyrus, the upper half of which was very narrow and of increased density. Posteriorly, this sclerosed gyrus was overhung and partially concealed by the adjoining superior parietal lobule. Apart from the distortion introduced by this apparent shrinkage and sclerosis the general convolutional patterning of the hemispheres exhibited no gross abnormality in the fissuring.

Microscopical Examination.-The following abnormalities were found in the representative areas of the central nervous system that have been examined (for an account of the areas selected and the methods used see Appendix).

1. The Meninges.-(a) The dura mater surrounding the patent part of the superior longitudinal sinus was normal. (b) The leptomeninges of the vertex of the brain showed simple fibroblastic thickening. The pial vessels were very engorged and some recent capillary hæmorrhages were seen.

2. The Cerebral Cortex.-(a) Extensive areas devoid of neurones were found in many of the cortical areas selected for examination. The macroscopically shrunken gyri showed this abnormality most obviously in the neighbourhood of the great longitudinal fissure. In this situation the whole grey matter cortex showed a severe diminution in the number of neurones, and both paracentral gyri exhibited areas of clearing in the third and fifth layers (fig. 1, A).

In the upper and middle portions of the precentral gyrus the neuronal defects were chiefly localized in the third or pyramidal cell-layer. The post- 
central gyrus and superior parietal lobule of the left hemisphere suffered in a similar way except that the granular layer ( $L$ iv) was equally affected. All

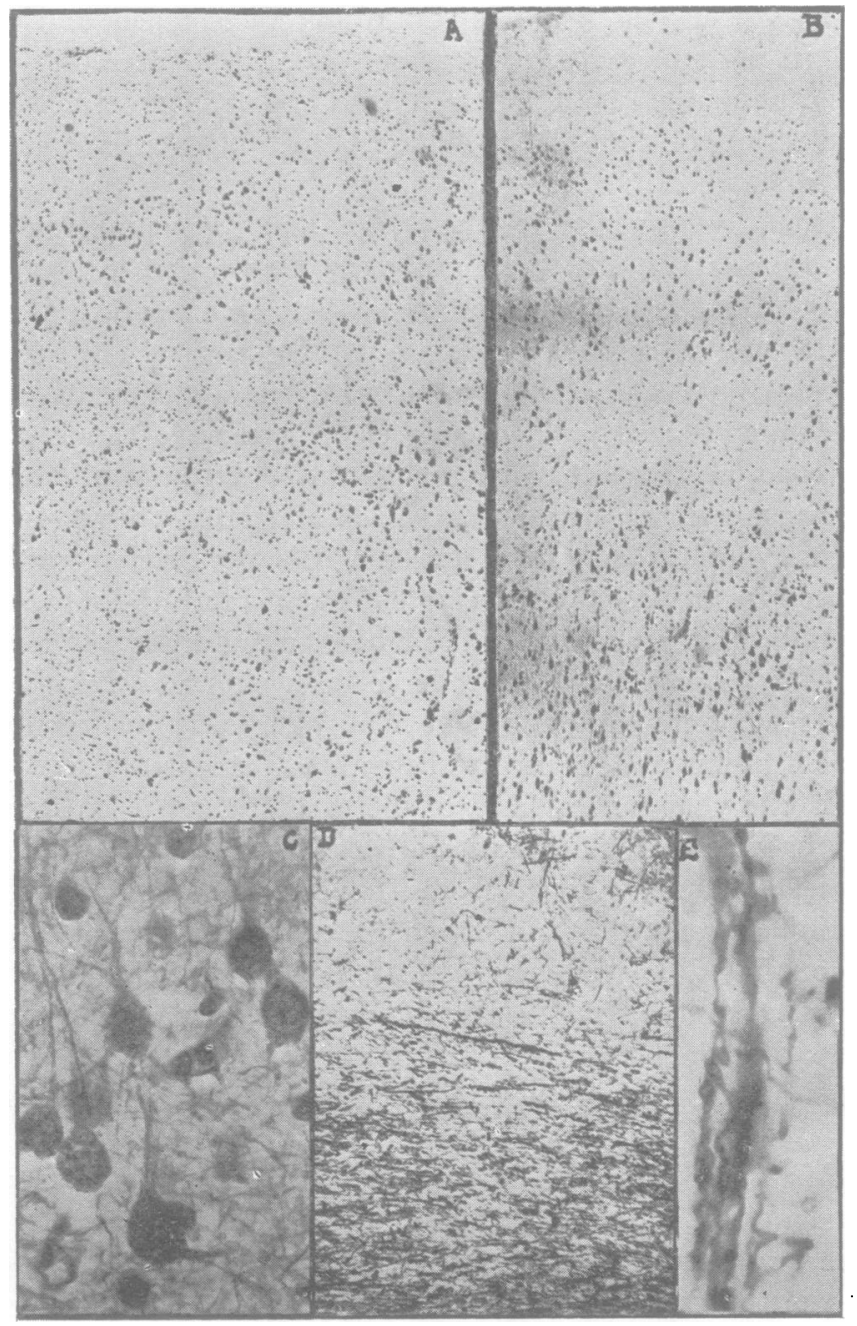

Fig. 1. A. Left paracentral lobule. Areas of cortical obliteration. Toluidin blue $\times 38$.

B. Left upper postcentral gyrus. Laminar defect in $L$ iii. Toluidin blue $\times 30$.

C. Primitive nerve-cells in wall of sclerotic precentral gyrus. Bielschowsky's stain $\times 475$.

D. Wall of postcentral gyrus. Anderson's modified Kulschitsky-Pal stain $\times 48$.

E. Wavy and beaded myelinated fibres in paracentral lobule. Stain as in D $\times 475$.

these cortical areas gave the appearance of being incompletely divided into two parts by an a-neuronal strip in the middle of the grey matter containing only an excess of glial nuclei (fig. 1, B). 
Smaller defects in the pyramidal cell-layer were found in other parts of the frontal lobe (FB, FC, FD), in the temporal lobe (TA, TG), and to a less

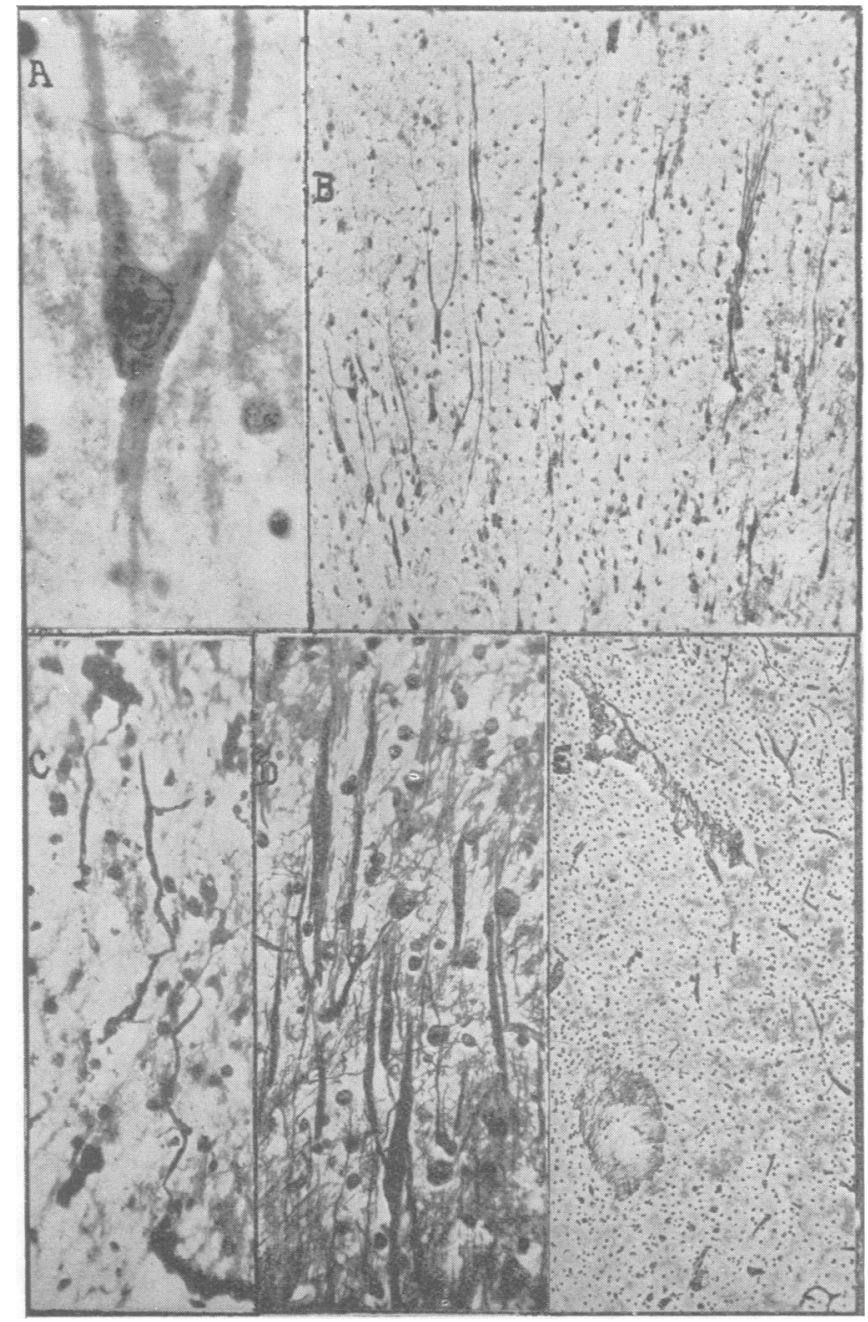

Fig. 2. A. Abnormal type of neurone from paracentral gyrus. Hortega's fourth variant of the tannic silver method $\times \mathbf{4 7 5}$.

B. Abnormal neurones in precentral gyrus. Cajal's gold sublimate method $\times 63$.

C. Mesenchymal reticulin fibrils in a sclerotic area of the cortex. Perdrau's method $\times 214$.

D. Swollen and agglutinated axiscylinders in paracentral gyrus. Bielschowsky's stain $\times 214$.

E. Fibrotic precapillary and mesenchymal proliferation in sclerotic white matter. Hortega's silver carbonate method for astrocytes $\times 63$.

degree in the visuopsychic area (OA). The frontalapole (FE) and the visuosensory area (OC) were intact. 
These neuronal defects were most frequently seen in the walls of the gyri, the crowns being unaffected save in the shrunken parts of the cortex. The nerve-cells in the deeper parts of the walls of the gyri were often represented as mere clusters or columns of small cells little better than neuroblasts. The Sommer sector of cornu ammonis showed a marked diminution in the cells of the pyramidal layer.

(b) Primitive and abnormal neurones were present in the abovementioned sclerotic parts of the cortex. Isolated clumps of nerve-cells which were developed little better than neuroblasts occurred chiefly in the deeper parts of the walls of the gyri (fig. 1, C). In addition to these primitive cells, peculiarly elongated or $\mathrm{Y}$-shaped forms were found in the proximity of the areas of cortical clearing (figs. 2, A, and 2, B). Such cells were not seen elsewhere in the cortex. In the sclerotic grey matter the axiscylinders occasionally exhibited thickening and agglutination reminiscent of senile changes (fig. 2, D).

(c) Glial changes.-There was marked sclerosis of the paracentral lobule, the upper pre- and postcentral gyri and adjoining superior parietal lobule. The overgrowth of fibrous neuroglia was patchily distributed between the remaining groups of nerve-cells and seemed to be denser around the bloodvessels (fig. $\mathbf{3}, \mathbf{D}$ ).

In the grey matter a laminar gliosis corresponding to the laminar neuronal outfall was often a conspicuous feature (fig. 3, A). The subpial felt work was thickened and strands of glial fibres were often seen passing between the surface of the gyrus and the deeper cortical layers. Here and there small cystic areas containing phagocytic cells were present in the more sclerosed gyri.

The white matter of the affected and neighbouring gyri and also the subjacent centrum ovale exhibited a pronounced gliosis of isomorphous type. Silver methods showed the number of astrocytes per field to be increased in the white matter of the less affected gyri (fig. 3, C). Examples of large fibre-forming macroglia were frequently demonstrated (fig. 3, B).

The oligodendroglia exhibited acute swelling with little intracytoplasmic content.

The microglia was present in normal forms in the unaffected parts of the cortex. In the small cystic areas mentioned above, compound granular corpuscles were present. An occasional elongated bipolar rod-cell was found in the frontal cortex adjoining the sclerotic areas, but there was no microglial mobilization.

(d) Changes in the myelin.-The radial and supraradial fibres in the sclerotic parts of the cortex were very poorly and patchily represented. The myelin sheaths exhibited numerous varicosities and their course was somcwhat wavy (fig. 1, E). In the postcentral gyrus a similar reduction in fibres had occurred, though even in this distorted area a few of the normal horizontal fibres of large calibre were demonstrable (fig. 1, D). No 'plaques fibromyéliniques' were seen. 
(e) Changes in the blood-vessels.-In the neighbourhood of the cortical areas characterized by the absence of nerve-cells the capillaries often showed

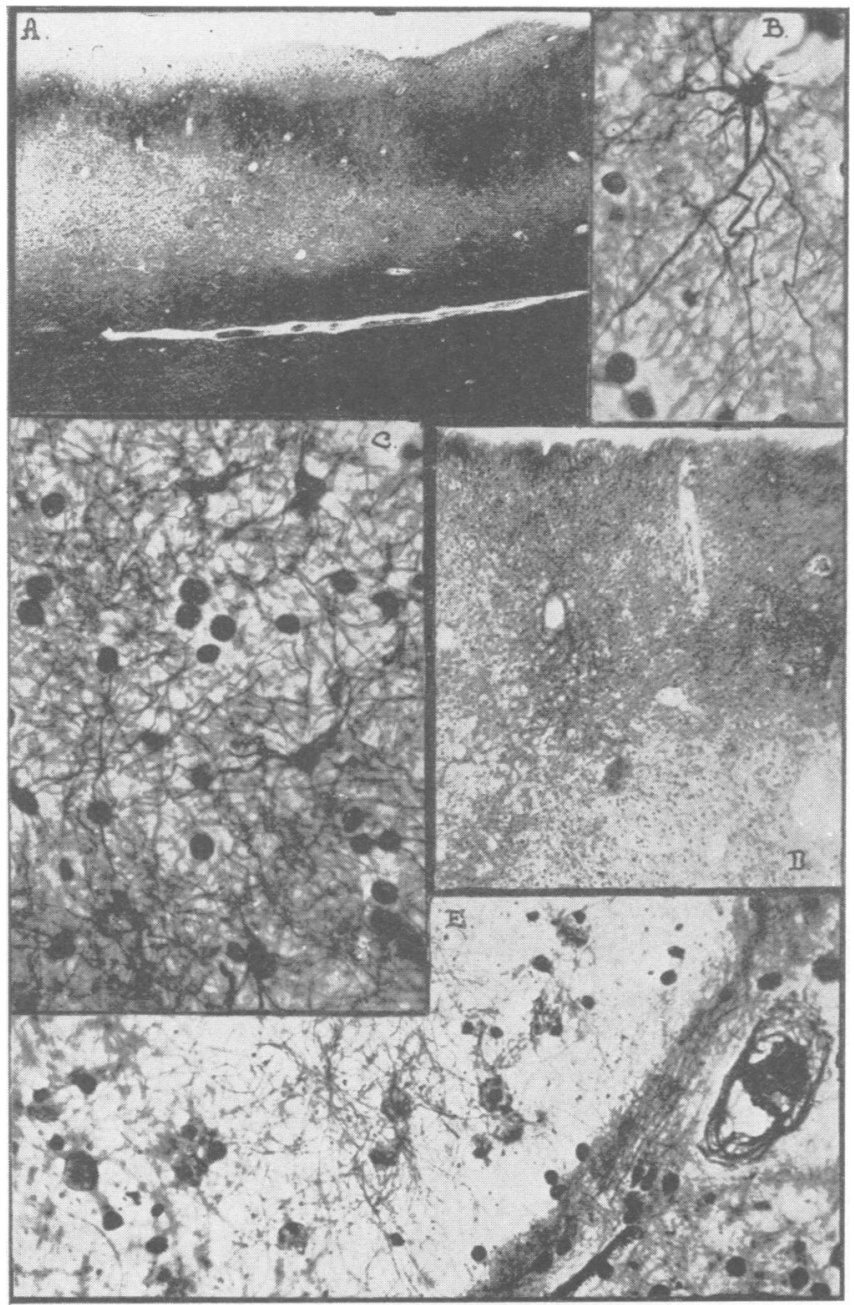

Fig. 3. A. Laminar gliosis in wall of postcentral gyrus. Holzer stain $\times 15$.

B. Type of large fibre-forming astrocyte found in less affected cortex (area FC). Hortega's method for astrocytes $\times 475$.

C. Gliosis in white matter of frontal cortex. Area and stain as in B $\times 214$.

I). Gliosis in paracentral gyrus. Holzer stain $\times 63$.

E. Paracentral gyrus. Phagocytic cells in cystic areas. Vessel at the right shows proliferation of mesenchymal fibrils. Stain as in $B \times 290$.

considerable fibrosis. Here also occurred an increase in visible vessels which was well demonstrated in sections stained by silver methods. In the areas of severe gliosis an overgrowth of the mesenchymal reticulin fibrils of the 


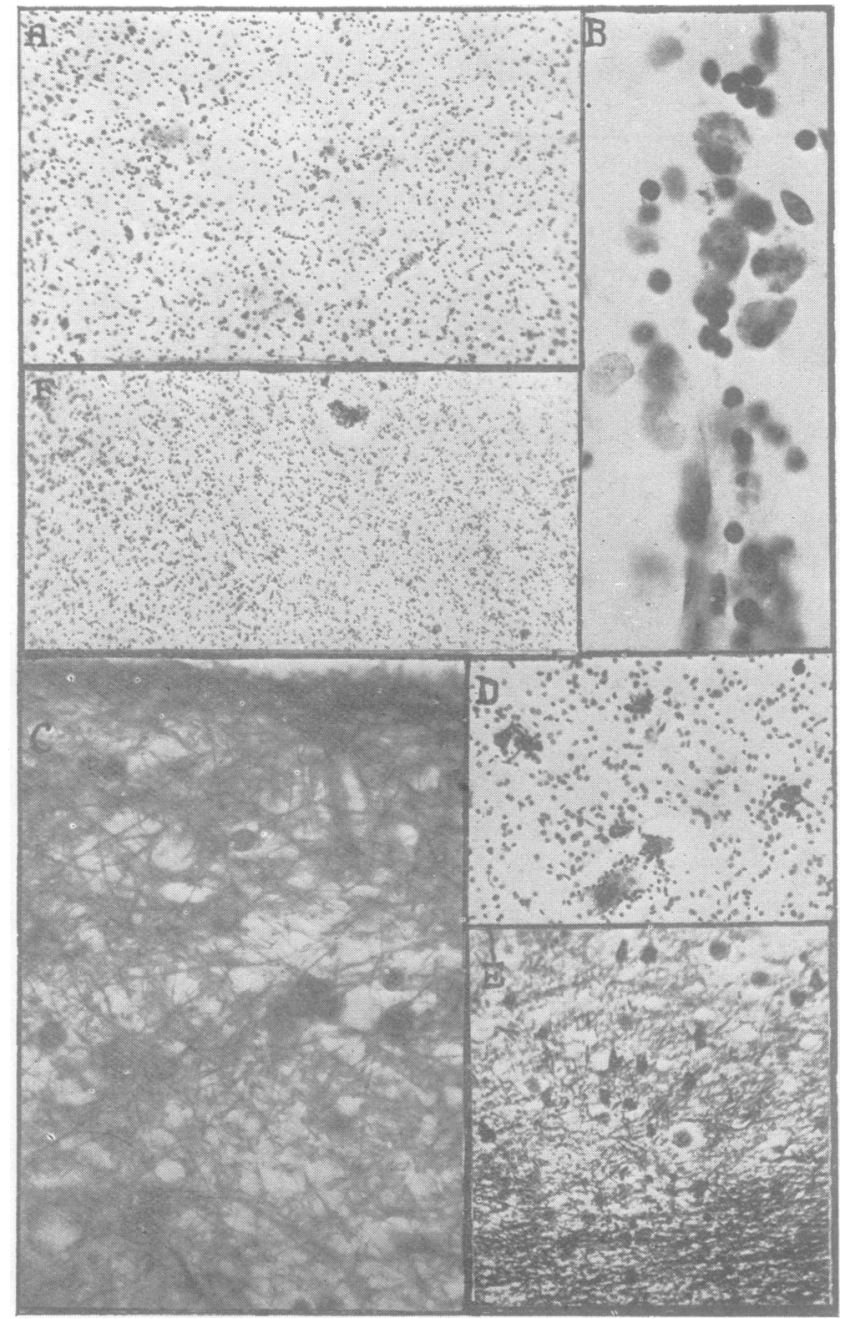

Fig. 4. A. Left putamen. Outfall of neurones and increase in glial nuclei. Toluidin blue $\times 48$.

B. Phagocytic cells in putamen. Toluidin blue $\times 475$.

C. Gliosis in putamen. Holzer stain $\times 475$.

D. Thalamus. Calcified neurones in lateral nucleus. Glial increase. Vessel with phagocytic cells. Toluidin blue $\times 63$.

E. Wall of precentral gyrus. Deposits of lipoid nature. Anderson's modified Kulschitsky-Pal stain $\times 79$.

F. Thalamus. Dense glial proliferation and gross reduction in neurones. Toluidin blue $\times 48$.

blood-vessels had occurred (figs. 2, C, and 2, E). Recent small capillary hæmorrhages were occasionally observed.

3. The Basal Ganglia.-(a) The thalamus showed an intense gliosis and a gross reduction in the number of neurones in the medial and lateral parts 
of the organ (fig. 4, F), the pulvinar and anterior nucleus being relatively well preserved. The nerve-cells remaining in these sclerosed areas were often

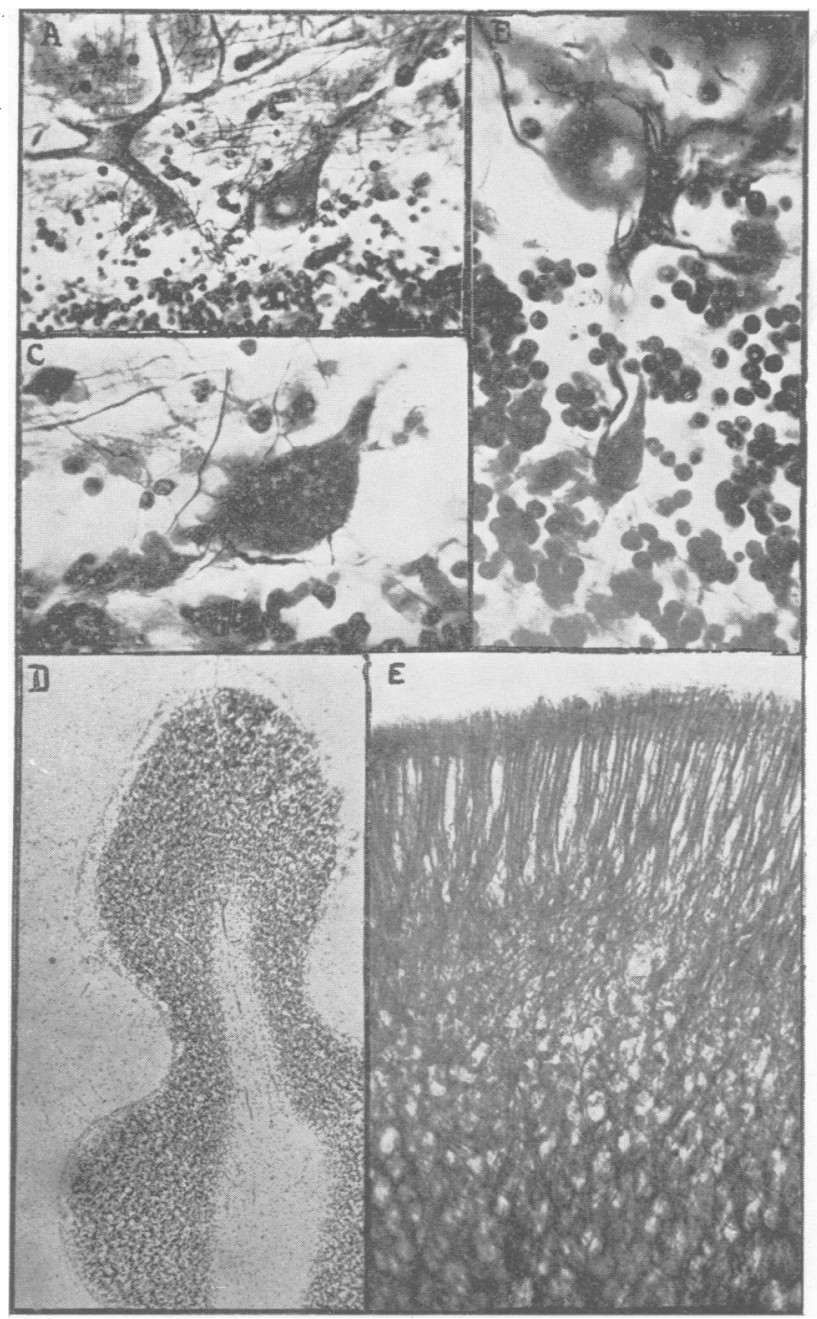

Fig. 5. A. Superior vermis of cerebellum. Hypertrophic antlering of the dendrites of Purkinje cell. Bielschowsky's stain $\times 214$.

B. As in A. Neuritic torpedo. Thickened basket network. $\times \mathbf{4 7 5}$.

C. As in $B$. $\times 475$.

D. As in A. Paucity of Purkinje cells. Increase in nuclei of Bergmann's glia. Toluidin blue $\times 29$.

E. Gliosis in superior vermis. Holzer stain $\times 214$.

calcified (fig. 4, D). The myelinated fibres in these affected areas were densely packed together. No status marmoratus was seen.

(b) The putamen also showed a diminution of nerve-cells and an over- 
growth of fibrous neuroglia (figs. 4, A, and 4, C). The vessel walls contained much fat and compound granular corpuscles were present in their vicinity

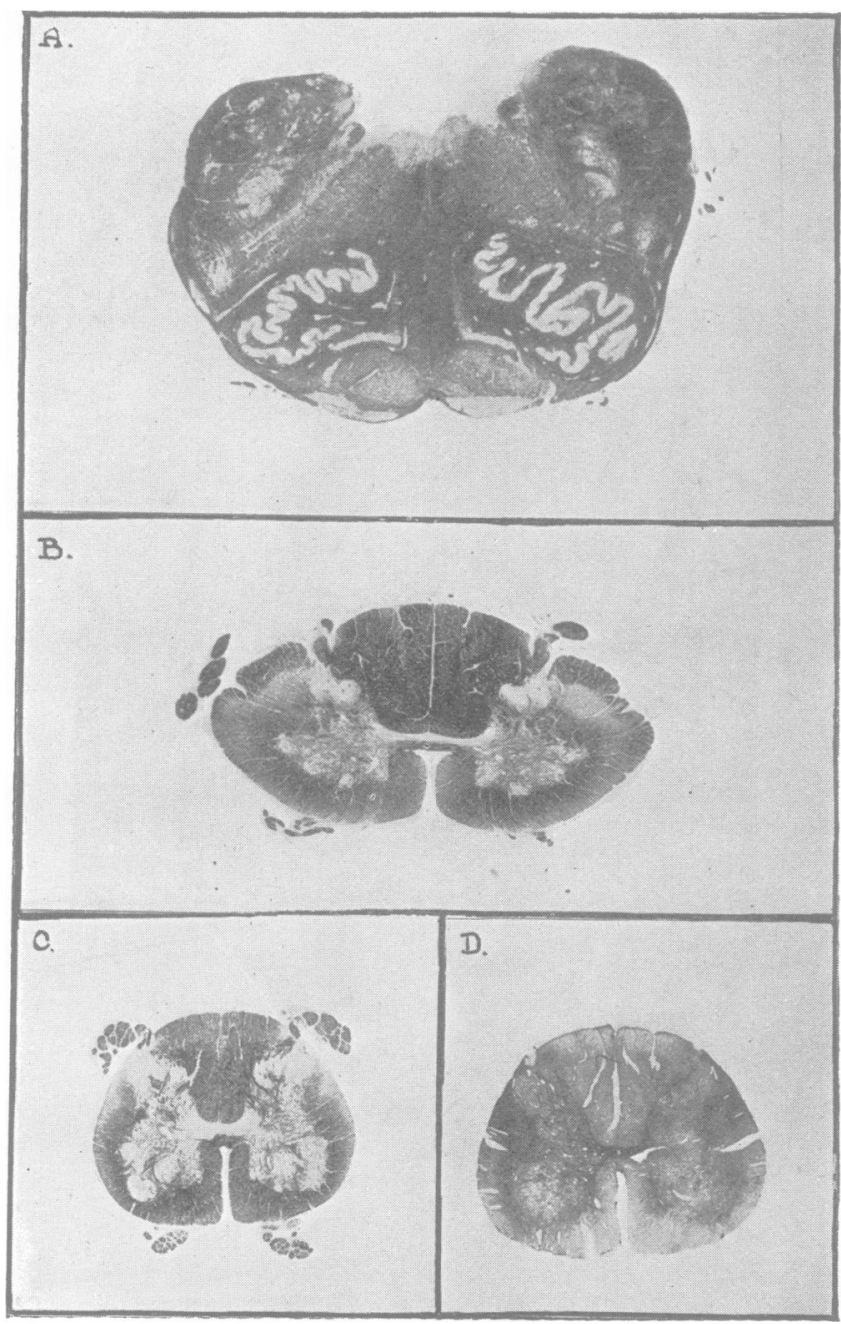

Fig. 6. A. Medulla oblongata. Degeneration of pyramids. Anderson's modified Kulschitsky-Pal stain $\times 3$.

B. Cervical enlargement. Degeneration of lateral columns. Stain and magnification as in $\mathrm{A}$.

C. Lumbar enlargement. As in $B . \times 3$.

D. Lumbar enlargement. Gliosis in lateral columns. Holzer stain $\times 3$.

(fig. 4, B). There were a few microscopic areas devoid of myelinated fibres and occasional coarse varicose fibres were present.

(c) The globus pallidus was normal apart from a slight crowding together 
of the nerve-cells. No abnormality in neurones, glia or myelin was found in either the head or body of the caudate nucleus.

4. The Cerebellum.--In sections prepared from the superior vermis it was obvious that some folia were very deficient in Purkinje cells (fig. 5, D). In these areas, which were the exception rather than the rule, the nuclei of the Bergmann glia-cells formed a distinct layer leaving a clear zone devoid of Purkinje cells in the subjacent parts of the folia. The radial glial fibres of the molecular layer were thickened and increased in number (fig. 5, E). Abnormalities were present in some of the Purkinje cells. These took the form of hypertrophy of the dendiitic antlers, hypertrophy of the basket networks and the presence of neuritic torpedoes (figs. 5, A, 5, B, and 5, C). The lateral lobes also showed a diminished number of Purkinje cells and glial overgrowth in some folia. In these parts the changes were less prominent than in the superior vermis. The central white matter of the cerebellum showed an excess of neuroglia-fibres and stained a darker shade with the Holzer method than did controls. Round the cells of the dentate nucleus there was a notable increase in glial nuclei. Myelinization of the central white matter appeared to be adequate.

5. The Condition of the Pyramidal Tract.-In the cerebral cortex the Betz cells appeared to be reduced in numbers owing to the defects present in the deeper layers of the paracentral lobules and upper precentral gyrus. Nevertheless, staining for myelin showed no obvious abnormality in the left internal capsule, crus or pons. At the level of the medullary olives the pyramids were smaller than normal and showed a definite thinning of myelinated fibres and an excess of glia (fig. 6, A). This defect in myelinization and coincident glial hyperplasia could be traced in the lateral columns down to the lumbar enlargement of the spinal cord (figs. 6, B, 6, C, and $6, \mathrm{D})$.

6. Products of Degeneration.- (a) Fat was present in excess in the vessel walls of the thalamus and putamen where phagocytic cells were present. The calcified nerve-cells of the thalamus showed numerous fatty globules adsorbed to their surfaces. The fatty granules present in the nerve-cells of the cerebral cortex appeared to be within normal limits.

(b) In the sclerotic parts of the cerebral cortex there were numerous small crumb-like deposits staining greyish-black with the Kulschitsky-Pal stain and resisting differentiation. They were confined to the deeper layers of the grey matter. They were not demonstrable by van Giesen staining and are thus not examples of ' colloid' degeneration (fig. 4, E).

(c) A large number of metachromatic granules were present in the internal capsule.

7. Capillary Homorrhages.-These were frequently seen in many parts of the nervous system. These bleedings were microscopic in size and consisted of unchanged red blood corpuscles. Slightly larger bleedings were present in the substantia reticulosa of the pons in the midline. 


\section{DISGUSSION}

Although the cortical changes described above are in the main typical of atrophic lobar sclerosis, ${ }^{12}$ the nerve-cell picture presented by those gyri showing extensive defects in the third layer might, perhaps, be confused with the four-layered type of cortex described by Bielschowsky in certain cases of microgyria. ${ }^{1}$ This latter condition, however, is a pure malformation brought about by the defective migration of neuroblasts from the matrix to the cortex. The neurones eventually become distributed in two main laminæ, a cell-free layer of myelinated fibres intervening between them. The superficial layer of these nerve-cells is usually arranged in a wavy or garland-like way (internal microgyria). The absence of fibrous gliosis in the grey matter of the cortex, the characteristic myelin picture and the grossly abnormal convolutional pattern of the brain make this type of microgyria readily distinguishable from the cortex in lobar sclerosis, where the abnormalities have been produced by a process of destruction, not of abnormal development. That such a destructive process has been operative in the present case is indicated by the dense gliosis in areas of the cortex devoid of neurones, the disturbance in the myelin picture, the presence of small groups of scavenger cells, and the proliferation of the mesenchymal fibrils of the blood-vessels. The arrested or perverted development of some of the remaining neurones in the affected areas is to be explained as the reaction on the part of immature cells to a grossly altered environment. In a like manner the extensive gliosis in the white matter may be attributed to the result of the stimulus to proliferation provided by disintegration products derived from foci of neuronal degeneration. Such findings are commonly found as sequelæ to lesions sustained by the immature nervous system during the period of anatomical plasticity.

The autopsy findings showed in the present case that the bilateral atrophic sclerosis of the cortex was associated with partial obliteration of the superior longitudinal sinus. According to Collier and Adie 4 " there is no other way out for the blood of the convexity of the hemispheres than by the superior longitudinal sinus, and it is for this reason that blocking of this sinus always results in disastrous bilateral softening of the hemispheres.' There is good reason for believing, therefore, that in this patient the cortical lesions have followed the sinus thrombosis, and, furthermore, since the child suffered from no sudden exacerbation of the general condition or from any illness in infancy, that the thrombosis did not occur later in life than the second day. The histological examination of the affected gyri showed that the severity of the destructive process diminished in a regular manner as the cortical areas adjoining the Sylvian fissure were approached until, finally, only small areas of neuronal obliteration were found in the lower parts of the walls of the convolutions. In other words, the parts of the brain most affected by the congestion and anoxæmia following the sinus thrombosis were those in 
which there was the least chance of the blood being led off by other channels than the superior longitudinal sinus-for example, by the inferior cerebral veins. The pathological process by which the neuronal destruction had been brought about was therefore somewhat similar to that claimed by Schwartz to be operative in some cases of birth injury to the brain, namely, vascular stasis following intense venous engorgement. It is significant that in the present case the layers of the cortex most affected ( $L$ iii and $L$ iv) were those in which the blood supply is normally the most abundant. It would seem that these neurones require the most abundant oxygen and that they suffer most by its deprivation.

The structural abnormalities found in parts of the nervous system remote from the cerebral cortex may be adequately accounted for as degenerative changes secondary to the primary cortical lesion. Biemond ${ }^{3}$ has demonstrated that atrophy of the cerebellar vermis may result from a lesion in the pons affecting the corticopontocerebellar fibres. The intimate connexion existing between the neopallium and cerebellum gives one good grounds for believing that the patchy atrophic change found in the cerebellum of the present case was secondary to the primary cortical destruction. Similarly, the remarkable poverty of neurones and the intense gliosis found in the medial and lateral parts of the thalamus are to be interpreted as secondary manifestations. A strictly comparable condition was found by Bielschowsky ${ }^{2}$ in his cases of cerebral hemiatrophy. Here the essential cortical lesion consisted in a widespread atrophy of neurones in the third layer of the cortex. He interpreted the areas of thalamic atrophy found in these brains as the result of a retrograde degeneration of the thalamocortical neurones consequent upon the destruction of the receptor layers of the cerebral cortex. The extensive involvement of the postcentral gyrus in the present case is strong evidence that a similar process was operative in producing a secondary atrophy of the thalamus. The comparative richness of the myelinated fibres in the atrophic subcortical areas is to be accounted for by the crowding together of the remaining axones owing to the shrinkage of the tissues.

The condition of the pyramidal tracts in this case deserves special mention, since the histological findings enable one to date roughly the onset of the cortical degeneration. It is necessary to reconcile two apparently contradictory facts revealed by microscopical examination. In the first place, no defect in myelinization of the pyramidal tracts was demonstrated in the pons, midbrain, or internal capsule. This observation does not support the view that a simple descending degeneration of the pyramidal fibres had followed destruction of the cells of origin in the infragranular cortex unless, indeed, the outfall of fibres was too limited to be clearly shown by the methods employed. Secondly, in the medulla and spinal cord there was present the typical picture of a demyelinization of the pyramidal tracts, namely, a diffuse outfall in myelinated fibres and an accompanying fibrous 
gliosis. The latter finding is evidence in favour of degeneration, not simply arrest of myelinization. An explanation of these apparently contradictory observations may be found when the distribution of the cortical lesions in this case is compared with that found in the variety of lobar sclerosis producing the remarkable condition of ' intracortical hemiplegia with intact pyramidal tracts.' In this type of cortical degeneration, several examples of which have been recorded in children and adults, ${ }^{2} 69101214$ a progressive atrophy of the pyramidal cells of the third cortical layer takes place, the infragranular cortex and the Betz cell-layer of the motor cortex remaining relatively intact. The result of this selective atrophy is that transcortical receptor impulses are prevented from reaching the effector projection-system by which voluntary movements are normally exteriorized. A clinical condition similar to ordinary hemiplegia is thus set up as this laminar degeneration of the cortex progresses, although no degeneration of the pyramidal tract is found owing to the fact that the cells of origin in the infragranular cortex are preserved. Now in the present case a similar laminar defect of the third layer of the cortex was present, but the peripheral portion of the pyramidal tracts exhibited degenerative changes. May it not be that this difference is due to the respective ages at which the atrophic cortical changes began ? In the children and adults in whom intracortical hemiplegia developed the pyramidal tracts had attained a degree of maturity which was not present in the case now being described. Normally, the pyramidal tract is incompletely myelinated at birth. This patient was a post-mature infant, so that it is reasonable to assume that myelinization had advanced somewhat more at the time of birth than would normally be the case. Nevertheless, the caudal part of the tracts would be still very imperfectly myelinated. Although an adult pyramidal tract is apparently able to withstand the abnormal situation resulting from the cessation of corticofugal impulses, it does not follow that incompletely myelinated fibres are equally robust. On these grounds, therefore, it seems probable that the degeneration of the pyramidal tracts in the present case was not a simple descending demyelinization, but rather an absorption of incompletely myelinized fibres resulting from a cessation of corticofugal impulses.

It follows from the above discussion that the time of onset of the thrombosis occurred not earlier than the end of fotal life-i.e. at a time when the pyramidal tract axones had penetrated the spinal cord but were still imperfectly myelinated. The birth history, as has been pointed out, suggested that an intracranial lesion had been sustained at birth and that an extension of the damage had occurred on the second day. Nevertheless, it is possible that this unusual accident may have been facilitated by prenatal factors. Apart from a developmental anomaly of the sinus itself, the possibility cannot be ruled out that the streptococcal infection of the mother during pregnancy may have acted as a predisposing influence in producing the thrombosis. The passage of cocci into the fotal circulation, their lodgement in the wall of 
the superior longitudinal sinus, and the ensuing toxic action upon the endothelium might undoubtedly form a focus for future thrombus formation.

Finally, it is readily understandable how the pathological changes in this brain have resulted in the clinical condition of idiocy. In the first place, the defect in the effector apparatus prevented the normal exploratory reactions of the infant; secondly, the lesions of the postcentral gyri shut out the kinæsthetic impulses the synthesis of which with exteroceptive sensory elements in the parietal association areas is the essential basis of cognition and behaviour. This latter consideration accounts for the lack of improvement to remedial training shown by the patient.

My thanks are due to Professor R. J. A. Berry, the Director of Medical Services at Stoke Park Colony, for permission to publish this case and for much helpful advice; to Dr. A. L. Taylor, Pathologist to Bristol General Hospital and to the Colony, with whom I have discussed many aspects of the work ; and to Dr. Cohn, who kindly supplied notes as to the neurological findings prior to the patient's admission to the Institution.

\section{APPENDIX}

\section{Areas Examined and Methods Employed}

The Cerebral Cortex. - With the exception of the right paracentral area, the left cerebral hemisphere only was examined. The following representative areas were studied : the upper. middle. and lower parts of the pre- and postcentral gyri ; the paracentral gyrus ; the frontal association areas (FB, FC, FD, FE of von Economo); the superior parietal lobule; the angular and supramarginal gyri ; the visuopsychic and visuosensory areas; the temporal pole and superior temporal gyri ; and the hippocampal convolution. Larger horizontal survey sections of the frontal and occipital lobes were also examined.

From the diencephalon of the left side sections have been prepared from the thalamus, putamen, globus pallidus, and head and body of the caudate nucleus, and also larger celloidin sections displaying the internal capsule in this situation.

The midbrain at the level of the emergence of the third cranial nerve, the medulla at the level of the middle of the olives and of the decussation of the pyramids, the upper cervical region and cervical enlargement, midthoracic and lumbar enlargement of the spinal cord have also been examined. lobes.

In the cerebellum sections have been studied from the superior vermis and lateral

The following staining methods have been adopted :

1. For Nissl's granules

2. For neurofibrils

3. For macroglia

toluidin blue.

Bielschowsky's stain.

Warkany-Holzer method, Cajal's gold sublimate and Hortega's silver carbonate method for astrocytes.

4. For oligodendroglia and microglia Hortega's method and the modification of Cone and Penfield.

5. For myelin

Anderson's modification of the Kulschitsky-Pal method, and osmic acid.

6. For fatty substances

Scharlach R. and Marchi's method.

Occasional use has also been made of Perdrau's modification of the Bielschowsky method and of Hortega's fourth variant of Achucarro's tannic silver process. 


\section{REFERENCES}

1 Bielschowsky, M., 'Über Microgyrie,'Jour. f. Psychol. u. Neurol., 1915, $22,1$.

2 Idem, ' Über Hemiplegie bei intakter Pyramidenbahn,' ibid., 1916, 22 (Erg.-Hft.).

3 Biemoxd, quoted by Brouwer in Localization of Function in the Cerebral Ccrtex, (Assoc. for Research in Nervous and Mental I)isease), 1934, 13, 8.

4 Collikr, J., and Adik, W. J., Price's Textbook of the Practice of Medicine, 4th ed., p. 1534 .

5 Dayton, N. A., Proc. 54th Annual Session of the American Association for the Study of the Feebleminded, 1930.

6 Freedon, L., 'Cerebral birth palsies. A contribution to their pathology with a report of a hitherto undescribed form,'Arch. Neurol. and Psychiat., 1931, 26, 524.

7 Gowers, W. R., A Manual of Diseases of the Nervous System, 2nd ed., 2, 461.

8 Hallevordex, J., 'Eigenartige und nicht rubrizierbare Prozesse,' Bumke's IIandbuch der Geisteskrankheiten, 1930, 11, 1096.

9 Hoestermaxw, E., 'Cerebrale Lähmung bei intakter Pyramidenbahn,' Arch. $f$. Psychiat., 49.

10 Јаков, A., 'Zum Capital der paradoxalen cerebralen Kinderlähmung,' Deut. Zeits. f. Nervenheilk., $1921,69$.

11 Rydberg, E., ' Cerebral injury in the new-born consequent on birth trauma ; with an inquiry into the normal and pathological anatomy of the neuroglia,'Acta path. et microbiol. Scand., Supplement. x, 1932.

12 Sснов, F., 'Pathologische Anatomie der Idiotie,' ref. as under (8) (p. 927).

13 Schwartz, Pr., 'Erkrankungen des Zentralnervensystems nach traumatischer Geburtsschädigung,' Zeits.f. d. g. Neurol. u. Psychiat., 1924, 90, 263.

14 Spielmeyer, W., 'Hemiplegie bei intakter Pyramidenbahn,' Münch. med. Woch., $1906,53,1404$.

15 Werthan, F. and F., The Brain as an Organ, 1934, 288. 